\title{
Jaynes-Cummings dynamics with a matter wave oscillator
}

\author{
Klaus Mølmer \\ QUANTOP, Danish National Research Foundation Center for Quantum Optics, \\ Department of Physics and Astronomy, University of Aarhus \\ DK-8000 Århus C, Denmark
}

\begin{abstract}
We propose to subject two Bose-Einstein condensates to a periodic potential, so that one condensate undergoes the Mott insulator transition to a state with precisely one atom per lattice site. We show that photoassociation of heteronuclear molecules within each lattice site is described by the quantum optical Jaynes-Cummings Hamiltonian. In analogy with studies of this Hamiltonian with cavity fields and trapped ions, we are thus able to engineer quantum optical states of atomic matter wave fields and we are able to reconstruct these states by quantum state tomography.

PACS numbers: 03.75.Gg, 03.75.Mn, 42.50.Fx
\end{abstract}

The Jaynes-Cummings model was introduced to describe the resonant interaction between an atom and a single mode of the quantized radiation field [1], and with incorporation of pumping and relaxation mechanisms, it constitutes the corner stone for the quantum treatment of maser and laser action 2. The unitary JaynesCummings dynamics has been succesfully demonstrated in the micro maser [3], in which atoms in Rydberg excited states are injected into a high-Q cavity with a single mode which is resonant with an atomic transition. This system has been used to demonstrate fundamental aspects of quantum theory such as the oscillatory exchange of energy between the atom and the field, sub-poissonian photon statistics, entanglement, and decoherence [4, 司. Another implementation of the Jaynes-Cummings Hamiltonian is in the description of the quantized motion of an ion in a harmonic trap [6], for which a classical laser field drives transitions in which the ion is excited and simultaneously one quantum of energy is extracted from the motional state. The ion system is characterized by very long lifetimes of the oscillator state, and hence the Jaynes-Cummings dynamics has been studied in great detail, and numerous examples of state synthesis and detection have been presented [7, 8].

In a quantum optical perspective, what characterizes a Bose-Einstein condensate is its single-mode character and coherence properties [9]. The bosonic commutator relation applies for atoms as well as for photons and phonons, and if one neglects interactions, many features of single mode fields are thus common for atoms, light and harmonic motion. This has of course led to various proposals for generation of atomic states with properties similar to the ones of light, e.g., phase and number squeezed states and Schrödinger cat states. By clever use of the atomic interactions as an adjustable non-linear element, strong quantum effects are foreseen [10], and in combination with the long storage times for atoms, these effects are potentially much more useful, for atoms than for light, and indispensable, e.g., for the improvement of atomic clocks 11 .

In this Letter we present a quantum optical analysis of the dynamics of a pair of atomic condensates which have been trapped in a periodic potential, so that one species has experienced the Mott insulator transition to a state with exactly one atom per lattice site 12, 13. The other condensate is assumed to have a different interaction strength, and hence it may still be in the superfluid phase with Poissonian number fluctuations on each site, or it may have reduced fluctuations due to the on site repulsion. A detailed presentation of this process was given recently [14]. When the optical lattice potential is raised the atomic wave functions become more localized in the potential minima, and thus the collisional interaction between atoms increases and the tunneling rate between wells decreases. From the time dependendent shape of the potential for the atoms one determines the on-site interaction strengths $U_{a}, U_{b}$ and $U_{a b}$ between atoms of the same and different species $A$ and $B$ and the interwell tunneling couplings $J_{a}, J_{b}$, but for simplicity we shall just assume the values shown in the upper panel of Fig.1. The ratio of approximately 0.6 between $U_{a}$ and $U_{b}$ corresponds to the ratio between the free space s-wave scattering lengths of $\mathrm{Rb}$ and $\mathrm{K}$ atoms. The tunneling coupling of both species are taken to follow the same lower curve. These curves are in qualitative agreement with the dependence of the interaction strength and tunnel coupling for a single species evaluated in [15], where the energy and frequency unit $J_{0}$, on the order of a tenth of the atomic recoil shift, is introduced $(\hbar=1)$. Using a symmetry breaking Gutzwiller ansatz, we assume that the full many body system is in a product state over all sites, where each site is described by the state vector $|\psi\rangle=\sum_{n_{A}, n_{B}} a_{n_{A}, n_{B}}\left|n_{A}, n_{B}\right\rangle$, with $n_{i}$ the number of atoms of species $i=A, B$. We have solved the selfconsistent equations for the amplitudes $a_{n_{A}, n_{B}}$, and we have verified that the two species undergo the Mott insulator transition at different times, see also 14]. The lower panel of Fig. 1. shows typical results of a numerical solution of the problem. The solid lines show the variance of the populations (the mean values are unchanged and equal to the initial variance where we assume Poisson statistics). The dashed curves are obtained by neglect- 
ing the cross coupling between the two species, and as in [14 we verify that this coupling has little effect on the population statistics of the two species in the lattice wells.

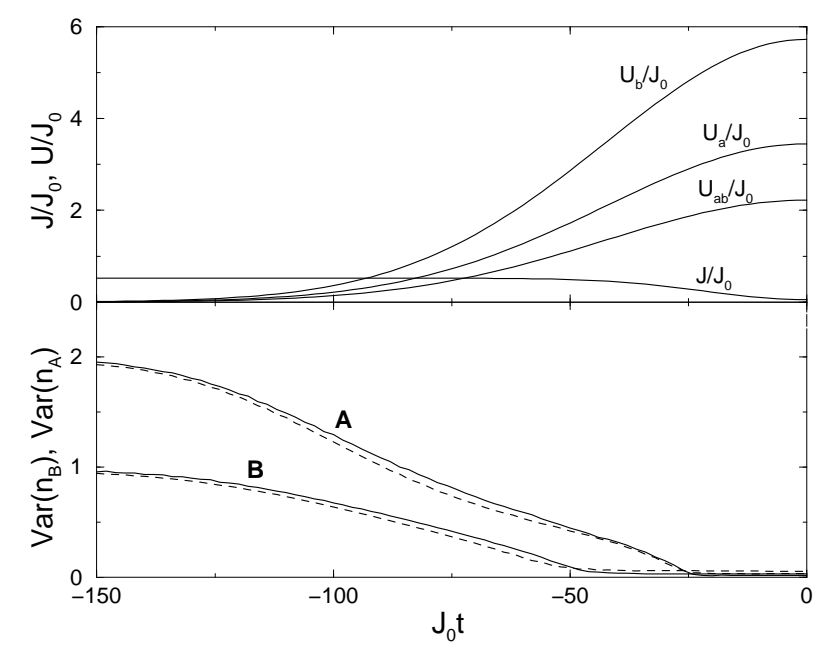

FIG. 1: Mott insulator transition for two atomic species. The upper panel shows the time dependent coupling constants and the tunneling coupling, all in units of the constant $J_{0}$. The lower panel shows the variance of populations of the two atomic species. The dashed curves are obtained by neglect of the cross coupling term $U_{a b}$. .

Numerous studies have addressed the prospects of forming molecules from ultra-cold atoms both theoretically and experimentally [16]. It has been suggested to trap the atoms in lattices with low occupation number, and either make explicit use of the Mott insulator transition 14, 15, or simply work with low mean occupation of each site 177 to form the molecules in an optimally controlled manner. We suggest to build on these proposals and to drive the photoassociation process on each lattice site to form a heteronuclear molecule with a single atom from each of the two species. We are more interested in the process than in the final molecular state, and hence a sufficiently long lived excited vibrational state will suffice as molecular state of the process. The coherent photoassociation process is described by the effective, second quantized, Hamitonian

$$
H=\chi a_{A} a_{B} a_{M}^{\dagger}+\chi^{*} a_{A}^{\dagger} a_{B}^{\dagger} a_{M}
$$

where $a_{i}$ is the annihilation operator of atoms and molecules $(i=A, B, M)$. When restricted to the states with initially precisely one $\mathrm{B}$ atom, we can rewrite this Hamiltonian as

$$
H=\chi a_{A} \sigma^{\dagger}+\chi^{*} a_{A}^{\dagger} \sigma
$$

where the Pauli spin lowering operator $\sigma=a_{B}^{\dagger} a_{M}$ represents the two-state transition operator $\left|1_{B}, \quad 0_{M}\right\rangle\left\langle 0_{B}, 1_{M}\right|, \quad(\sigma$ turns an $\mathrm{AB}$ molecule into a $\mathrm{B}$ atom, i.e. , it does not conserve the number of $\mathrm{A}$ atoms, and it appears only in combination with the creation operator $a_{A}^{\dagger}$ ). The Hamiltonian couples pairs of levels

$$
\left|n_{A}, 1_{B}, 0_{M}\right\rangle \leftrightarrow\left|(n-1)_{A}, 0_{B} 1_{M}\right\rangle,
$$

and it thus follows that there will be an oscillatory exchange of the single $\mathrm{B}$ atom between being part of the molecule and being an independent atom, in complete analogy with the Jaynes-Cummings transfer of excitation between a two-level system and an oscillator mode.

This oscillation will undergo collapses and revivals, because the coupling strength betwen the two mentioned states is proportional to $\sqrt{n_{A}}$, and hence the different number state components of $\mathrm{A}$ atoms will give away an atom to the association process at different frequencies. An experiment that counts the total number of B atoms as a function of duration of the photoassication process, can thus teach us about the number statistics of the A atoms!

So far, the Mott insulator transition has been demonstrated experimentally by the loss of interference between different lattice sites, and by a gap in the excitation spectrum [13]. The Jaynes-Cummings dynamics presents a unique application of a quantum optical method for the study of this many-body problem. We suggest to turn on slowly the lattice potential, so that the B atoms undergo the transition to the Mott phase at a time where the $\mathrm{A}$ atoms are still in an unknown state. At this time we hold the lattice potential and we turn on the photoassociation process, i.e., the dynamics driven by the Jaynes-Cummings Hamiltonian (2). The probability to find a $\mathrm{B}$ atom on any lattice site is given by the square of the Rabi oscillation amplitudes, weighted by the $n_{A}$ probability distribution $p\left(n_{A}\right)$ :

$$
\left\langle n_{B}\right\rangle=\sum_{n_{A}} p\left(n_{A}\right) \cos ^{2}\left(\chi \sqrt{n_{A}} t\right)
$$

This function is illustrated in Fig. 2. which shows the mean value of $n_{B}$ as a function of time in case of coupling to a Poissonian distribution with on average two A atoms per lattice site (upper panel), a sub-Poissonian distribution of A atoms achieved at $J_{0} t=-50$, cf. Fig 1 , during application of the periodic potential (middle panel), and an $n_{A}=2$ (Mott insulator) number state (lower panel). For time sequences like the ones in Fig.2, one can use Eq.(㺼) to obtain a fit, which produces the complete $p\left(n_{A}\right)$ distribution. By counting the total number of B atoms, without requiring the experimental ability to detect atoms with high efficiency on a microscopic spatial scale, we thus obtain the precise number statistics at each site of the A atoms. A similar procedure was initially applied for trapped ions [7], where the internal state of the ion was used to monitor the population of the harmonic oscillator levels of motion. Many repeated 
experiments on the same system were necessary to probe the state of the ion at different durations $t$ of the dynamics and with good precision. We also have to operate the process many times with different durations of the photoassociation process, but since the experiment 'repeats itself' in parallel in every lattice site, determination of the total number of B atoms in the entire system suffices to give the mean value of $n_{B}$. To take into account both deviations from the Lamb-Dicke limit and decoherence, in Ref. [7] the ideal Jaynes-Cummings dynamics (14) was replaced by a more general formula

$$
\left\langle n_{B}\right\rangle=\sum_{n_{A}} p\left(n_{A}\right) \cos ^{2}\left(\Omega_{n_{A}} t\right) e^{-\gamma_{n_{A}} t}
$$

with fitting parameters $p\left(n_{A}\right), \Omega_{n_{A}}$ and $\gamma_{n_{A}}$. Similar extra handles on the theory may be useful in our matterwave analog where, e.g., atom-atom interactions may cause an $n_{A}$-dependence of the atomic wave function and hence of the photo-association coupling rate, and where various mechanisms, such as inhomogeneities over the size of the lattice, may cause decoherence.

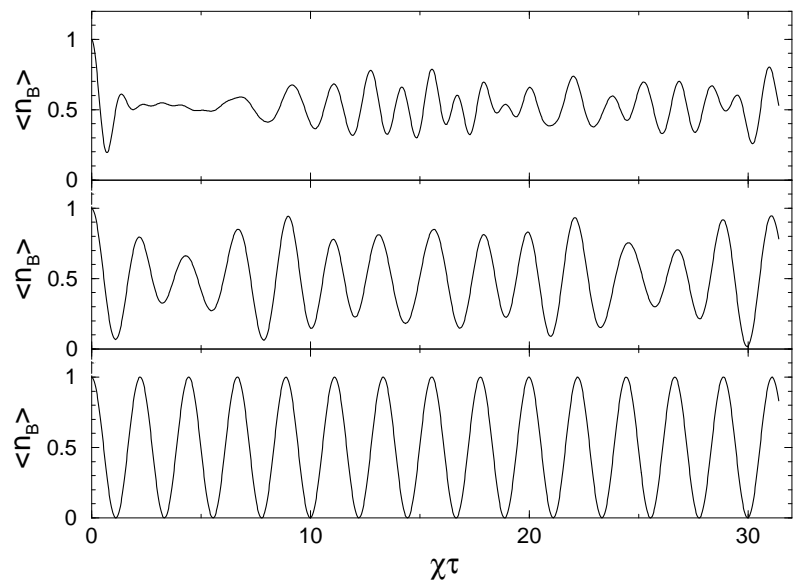

FIG. 2: Time dependence of the mean number of B atoms per lattice site during photoassociation/-dissociation. The upper panel shows the results for a Poissonian distribution with an average of two A atoms per site; the middle panel shows the results for the distribution of $\mathrm{A}$ atoms at $J_{0} t=-50$ in Fig. 1 , where the Mott insulator transition has not yet taken fully place for the A atoms; and, the lower panel shows the results for an $n_{A}=2$ number state.

In case one can only effectively control the Mottinsulator transition of one species (the A atoms), we note that for a sufficiently low density of B atoms, every lattice site will be occupied by a single or none of these atoms, and hence the mean value of $n_{B}$ will ocillate in the same way as in (4,5) but multiplied with the mean number of $B$ atoms per site.

As mentioned in the introduction of the Letter, the Jaynes-Cummings Hamiltonian has been applied extensively in quantum optics, and the range of interesting phenomena studied is very wide. Let us turn to the possibilities for synthesis and studies of particular quantum states of matter by means of the effective Jaynes-Cummings Hamiltonian. Studies of the collapse and revival of the diffraction pattern of lattice trapped atoms have already been made [19, 20, and we propose to use the Jaynes-Cummings dynamics to produce a Schrödinger cat like state of the A atoms, and to probe its coherence properties. The photoassociation process can be driven off resonance with a detuning $\delta$ in which case the two-state transition (3) does not take place, but the coupling (2) perturbs the energy of the state $\left|n_{A}, 1_{B}\right\rangle$ by the amount $\Delta E_{n_{A}}=|\chi|^{2} n_{A} / \delta$. If the $\mathrm{A}$ atoms are in a coherent state $|\alpha\rangle$, this state will undergo a simple phase rotation, $|\alpha\rangle \rightarrow\left|\alpha e^{i \phi}\right\rangle$ due to this coupling. Imagine now that the $\mathrm{B}$ atom has a level structure, so that it can be initally prepared in a superpostion of the active state $|B\rangle$ which experiences the coupling discussed above, and a passive state $\left|B^{\prime}\right\rangle$ for which the photoassociative coupling vanishes completely. This implies that the Jaynes-Cummings Hamiltonian produces a material Schrödinger cat like state of the two species,

$$
|\Psi\rangle=a\left|\alpha e^{i \phi}\right\rangle \otimes|B\rangle+b|\alpha\rangle \otimes\left|B^{\prime}\right\rangle,
$$

very analogous to the production of a similar state in cavity QED experiments [5]. By driving the transition between the active and the passive level in the B atom, we can subsequently monitor the interference of the two coherent components. The production of a mesoscopic superposition state can thus be verified experimentally. In a gauge invariant formulation of the problem, there is of course not a coherent state and mean atomic field in a single site, but a total number state of the entire atomic ensemble may be well approximated by a coherent state, and when this is split into a product of coherent states on each lattice site, a total number projection operator produces a very entangled state of the atomic components on each site [17], but physical observables will be correctly handled by the coherent state ansatz [18].

The cavity QED implementation of the JaynesCummings Hamiltonian allows for classical excitation of both the two-level system and the oscillator, and it operates experimentally with the injection of a beam of excited two-level atoms, who can give off their excitation energy to the oscillator degree of freedom. In the ion trap implementation, both the oscillator and the two-level system can be excited independently, and by changing the coupling frequency an "anti" Jaynes-Cummings Hamiltonian $\chi a \sigma+\chi^{*} a^{\dagger} \sigma^{\dagger}$, and a "two-phonon" Hamiltonian $\chi a^{2}+\chi^{*}\left(a^{\dagger}\right)^{2}$ can be implemented to produce various non-classical states [7]. Tomographic reconstruction of the motion of a trapped ion has been demonstrated in [8]. That measurement scheme made use of a classical driving to displace the oscillator by the complex amplitude $\alpha$ and subsequently the populations of the various oscillator states were probed by the two-level population oscillations (4.5) as described above. The position-momentum 
Wigner function was then obtained by an analytical formula [8] from the populations recorded with different displacement $\alpha$.

It will add an experimental level of difficulty to couple atoms coherently from a reservoir condensate into the lattice potentials, but with the current progress in transfer and loading of degenerate gasses into microfabricated structures [21], some possibilities are certainly open. Without continuous loading of the lattice, there is another possibility to mimic the quantum optical effects driven by external, classical fields. We illustrate this with a protocol for full quantum state tomography of the matter wave field of the A atoms: Since we cannot accomplish coherent displacements of the atomic field we suggest to use A atoms with two internal states, the active state $|A\rangle$ and a passive state $\left|A^{\prime}\right\rangle$ which is not affected by the photoassication process. We can then use the $\left|A^{\prime}\right\rangle$ population as a 'local oscillator', and as long as the A'-population is large, coherent transfer of atoms between the two atomic states is well described by a coherent displacement of the A atom oscillator. In fact, this internal state coupling is correctly described by means of a rotation of the Dicke collective spin of the atoms, and the detection of the population statistics of the $|A\rangle$-state atoms by means of the Jaynes-Cummings dynamics can be used for perfect collective spin tomography: If there is a total of $N$ atoms populating states $|A\rangle$ and $\left|A^{\prime}\right\rangle$ in every lattice site, it takes $4 N+1$ effective spin rotations followed by population measurements to reconstruct the full collective spin density matrix, following the protocol applied to large hyperfine manifolds in Ref. 22.

In summary, we have suggested how to realize a purely atomic Jaynes-Cummings Hamiltonian and how to use it both to generate quantum optical states of matter and to study various many-body properties of the system. To isolate the different effects and mechanisms, we did not present studies of the joint effect of interactions, inter-well tunneling and the photoassociation process. It is clear that these effects in combination may provide wide possibilities for detection and production of quantum states. It has been estimated in 11, 15] that one can reliably transfer atoms coherently between the trapped ground states of the system, but it is of course also an interesting possibility to use the resonance conditions for the photoassociation lasers to excite and study collective excitations of the atoms. We have focussed on lattice trapped atoms, where the coherent Jaynes-Cummings dynamics is most readily realized, and where the existence of many replica of the same quantum systems provides effective means for detection of generic quantum optical behavior of the matter wave fields. Within this framework, one can also consider multiple species and multi-level systems in analogy with multi-mode and multi-level generalizations of the JaynesCummings Hamiltonian. The dynamics is not restricted to lattices, and as an extension to the work we imagine that a collection of distinguishable, e.g., spatially localized $\mathrm{B}$ atoms immersed in a larger trapped condensates of A atoms can serve to induce a host of interesting effects in analogy with the more general interaction of light and two-level atoms. An appealing possibility would, for example, be to look for a matter wave analogue of the Mollow fluorescence triplet, when an atomic laser beam interacts with an ensemble of B atoms and molecules can dissociate to atomic states with higher and lower energy than the energy of the incident beam.

Comments on the manuscript by Michael Budde are gratefully acknowledged.

[1] F. W. Cummings, Phys. Rev. 140, A1051 (1965).

[2] M. Sargent III, M. O. Scully and W. E. Lamb, Jr, Laser Physics, Addison-Wesley, Reading, Massachussetts, (1974).

[3] D. Meschede et al, Phys. Rev. Lett. 54, 551 (1985); P. Filipowicz et al, Phys. Rev. A 34, 3077 (1986).

[4] G. Rempe et al, Phys. Rev. Lett. 58, 353 (1987); M. G. Raizen et al, Phys. Rev. Lett. 63, 240 (1989); M. Brune et al, Phys. Rev. Lett. 65, 976 (1990).

[5] M. Brune et al, Phys. Rev. Lett. 77, 4887 (1996).

[6] J. I. Cirac, et al, Phys. Rev. Lett. 70, 762 (1993).

[7] D. M. Meekhof, et al, Phys. Rev. Lett. 76, 1796 (1996).

[8] D. Leibfried, et al, Phys. Rev. Lett. 77, 4281 (1996).

[9] M. Kasevich, Science 298, 136, november (2002).

[10] A. Sørensen et al, Nature 409, 63 (2001).

[11] G. Santarelli, et al, Phys. Rev. Lett. 82, 4619 (1999).

[12] D. Jaksch et al., Phys. Rev. Lett. 81, 3108 (1998).

[13] M. Greiner, et al, Nature, 415, 39-44 (2002).

[14] B. Damski et al, cond-mat/0208375.

[15] D. Jaksch, et al., Phys, Rev. Lett. 89, 040402 (2002).

[16] R. Wynar, et al, Science 287, 1016 (2000); M. Mackie et al., Phys. Rev. Lett. 84, 3803 (2000); J. Javanainen and M. Mackie, Phys. Rev. Lett. 88, 90403 (2002); C. McKenzie et al. Phys. Rev. Lett. 88, 120403 (2002): S.J.J.M.F. Kokkelmans, and M.J. Holland, condmat/0204504: T. Koehler, T. Gasenzer, and K. Burnett, cond-mat/0209100; E. Donley et al, Nature 417, 529 (2002).

[17] T. Esslinger and K. Mølmer, cond-mat/0210324.

[18] K. Mølmer, Phys. Rev. A 55, 3195 (1997); J. Mod. Opt. 44, 1937 (1997).

[19] M. Greiner et al, Nature, 419, 51-54 (2002).

[20] C. Orzel et al., Science 291, 2386 (2001).

[21] W. Hänsel et al, Nature 413, 498 (2001); H. Ott, et al, Phys. Rev. Lett. 87, 230401 (2001); A. E. Leanhardt, Phys. Rev. Lett. 89, 040401 (2002).

[22] G. Klose et al, Phys. Rev. Lett. 86, 4721, (2001). 